\title{
Zur Einbettung von Foren deliberativer Demokratie in repräsentative Institutionen
}

Sabine Kropp

\section{Einführung: Eine ungebrochene Popularität deliberativer Demokratie?}

In den vergangenen Jahren sind in westlichen Demokratien zahlreiche Foren, Dialoge, Mediationsverfahren und sog. »Mini-Publics « ${ }^{1}$ eingerichtet worden, um die Zivilgesellschaft stärker in Entscheidungsprozesse einzubeziehen und repräsentativen Institutionen durch eine breitere Partizipation eine höhere Legitimation zu verleihen. Im Wesentlichen zielen solche deliberativen Elemente auf eine Ergänzung von Verfahren, die in den Rahmen repräsentativer Institutionen eingebettet sind. Foren deliberativer Demokratie sind mittlerweile auf allen Ebenen und in zahlreichen Politikfeldern zu finden - neben den in anderen Kapiteln dieses Buches im Vordergrund stehenden Verfassungsprozessen z.B. im Bereich der Infrastrukturpolitik (Stromnetzdialoge), zur Befriedung lokaler Nutzungskonflikte $^{2}$ oder als von der Regierung initiierter, anschließend teilweise von gesellschaftlichen Organisationen selbst durchgeführter und wissenschaftlich begleiteter Prozess, in dem die Bürger in verschiedenen Politikfeldern ihre Vorstellungen von einem "guten « Leben diskutieren sollen. ${ }^{3}$ Manche Untersuchungen heben die deliberative Qualität des Schweizerischen "Vernehmlassungsverfahrens", insbesondere in den italienischsprachigen Kantonen hervor, ${ }^{4}$ andere wiederum sehen auch in der Komitologie der

1 Vgl. hierzu Kimmo Grönlund, André Bächtiger und Maija Setälä (Hrsg.), Deliberative Mini-Publics: Involving Citizens in the Democratic Process (ECPR Press, 2014).

2 Vgl. z.B. den sog. Berliner »Bello-Dialog« zur Ausarbeitung eines Hundegesetzes, www.berlin.de/sen/verbraucherschutz/aufgaben/tierschutz/hundehaltung/bello-dia log-541321.php (abgerufen am 21. November 2017).

3 www.gut-leben-in-deutschland.de/static/LB/index.html (abgerufen am 2. November 2017).

4 Stefan Neubert, »Kantonale Vernehmlassungsverfahren im Vergleich«, (2008) 2 LeGes 271; Thomas Sägesser, »The Consultation Procedure in Switzerland «, (2004) 3 LeGes 113. 
EU-Kommission deliberative Elemente verwirklicht. ${ }^{5}$ Einige Autoren weisen wiederum darauf hin, dass eine Massendeliberation bessere und repräsentativere Ergebnisse erzeuge als eine Debatte in kleineren Foren mit weniger Teilnehmern. ${ }^{6}$

Allein die große Zahl an solchen - höchst unterschiedlichen - Foren wirft indessen die Frage auf, welche Funktion ihnen in Entscheidungsprozessen zugedacht wird, welche Bedeutung ihnen in der Realität zukommt, und welche realen Effekte sie im Institutionensystem erzeugen. Einschränkend sei vorausgeschickt, dass bei Weitem nicht alle diese Foren den strengen Maßstäben deliberativer Demokratie genügen, auch wenn sie im weiteren Sinne solchen Partizipationsformen zugerechnet werden. Die Erscheinungsformen deliberativer Demokratie und die Art und Weise, wie solche Foren durchgeführt werden, sind so vielfältig, dass ihre systematische Erfassung und vollständige Auswertung unmöglich sind. Die nachfolgende Darstellung nimmt angesichts dessen einige inhaltliche Einschränkungen vor. Sie konzentriert sich mit Blick auf die thematische Ausrichtung des vorliegenden Bandes auf solche Ansätze, die ein partizipatorisches Anliegen verfolgen, d.h. in emanzipatorischer Stoßrichtung "mehr» Demokratie realisieren möchten, ${ }^{7}$ und diskutiert abschließend, inwiefern die in der Theorie strengen Maßstäbe an die Verfahrensgestaltung für eine Bewertung real existierender deliberativer Foren geeignet sind. Der Text blendet im Folgenden die epistemisch ausgerichteten, wissensbasierten Formen der Politikgestaltung weitgehend aus, da sie auf ein anderes Untersuchungsfeld führen - auf das der Wechselbeziehungen zwischen Politik und Wissenschaft.

5 Annette E. Töller, Komitologie: Theoretische Bedeutung und praktische Arbeitsweise von Durchführungsausschüssen in der Europäischen Union am Beispiel der Umweltpolitik (Budrich, 2002).

6 Vgl. James S. Fishkin und Robert C. Luskin, »Experimenting with a Democratic Ideal: Deliberative Polling and Public Opinion«, (2005) 40 Acta Politica 284; Marlène Gerber und Sean Müller, »When the People Speak - and Decide: Deliberation and Direct Democracy in the Citizen Assembly of Glarus, Switzerland «, (2018) 43(3) Policy \& Politics 371-390.

7 Vgl. grundlegend: Jürgen Habermas, Strukturwandel der Öffentlichkeit: Untersuchungen zu einer Kategorie der bürgerlichen Gesellschaft (Suhrkamp, 1990); Jürgen Habermas, Faktizität und Geltung: Beiträge zur Diskurstheorie des Rechts und des demokratischen Rechtsstaats (4. Aufl., Suhrkamp, 1994); Jürgen Habermas, Die Einbeziehung des Anderen: Studien zur politischen Theorie (Suhrkamp, 1999). 
Zunächst werden einige zentrale Grundideen deliberativer Demokratie knapp zusammengefasst. ${ }^{8}$ Da der Literaturstand hierzu breit aufgefächert ist und hier keine erneute Zusammenfassung des Forschungsstands erfolgen kann, konzentriert sich der nachfolgende Beitrag zuvorderst darauf, einige institutionelle Implikationen zu erörtern, die sich aus der Anwendung deliberativer Elemente ergeben. Dabei werden insbesondere Problematiken dargestellt, die durch die Einbeziehung partizipativer Bausteine in Mehrebenensystemen sowie durch die zeitliche Taktung verschiedener Partizipationsformen entstehen. Die Herausforderungen partizipativer Demokratie variieren selbstredend mit der real existierenden Vielfalt von Institutionenordnungen. Als empirische Referenzpunkte dienen im Folgenden die Erfahrungen in Deutschland und, um diese zu kontrastieren, ansatzweise in der Schweiz. Auf dieser Grundlage schließt der Beitrag mit einem Ausblick auf den möglichen Mehrwert deliberativer Demokratie für die Gestaltung repräsentativer Institutionen. Er belegt, dass der hohe normative Anspruch der Theorie weitgehend unerfüllbar bleiben muss und deliberative Demokratie somit als eine Art regulativer Idee bzw. als rekonstruktives Verfahren begriffen werden sollte, das rationale Entscheidungsprozesse jenseits des empirisch Erfahrbaren verdeutlichen kann. Der Beitrag schließt mit dem Befund, dass Deliberation durchaus die Legitimationsbasis von Politik verbessern kann; ihr Einbau in das repräsentative Institutionensystem und in die Gesamtheit bestehender Partizipationskanäle ist dabei jedoch an spezifische Bedingungen und die Notwendigkeit von Folgenabschätzungen geknüpft.

\section{Ansprüche deliberativer Demokratie}

Konzepte deliberativer Demokratie, insbesondere in ihrer beteiligungszentrierten, emanzipatorischen Variante, ${ }^{9}$ sind als normative Theorien zu begreifen. Sie gehen davon aus, dass die Bürger zu mehr Demokratie und zu einer besseren Beteiligung grundsätzlich befähigt sind. ${ }^{10}$ Trotz ihrer ungebrochenen, langanhaltenden Karriere sind diese Theorien erfahrungswissenschaftlich aber nur wenig abgesichert, auch wenn dieses Defizit

8 Vgl. ausführlich die Diskussion in: Oliver Flügel-Martinsen et al. (Hrsg.), Deliberative Kritik - Kritik der Deliberation: Festschrift für Rainer Schmalz-Bruns (Springer, 2014).

9 So insbesondere bei Jürgen Habermas.

10 Manfred G. Schmidt, Demokratietheorien: Eine Einführung (5. Aufl., Springer, 2010), 240 . 
allmählich durch empirisch fundierte Beiträge ausgeglichen wird. ${ }^{11}$ Die wesentlichen Ziele der Theorie sind darauf gerichtet, eine umfassende gesellschaftliche Inklusion und breite Partizipation zu begründen. Dies soll durch eine abwägende, verständigungsorientierte Beratschlagung erfolgen, ${ }^{12}$ wobei die demokratische Legitimität von der prinzipiell möglichen - nicht immer faktischen - Zustimmung der Bürger auf der Grundlage eines diskursiven und partizipativen Prozesses abhängt. ${ }^{13}$

Die grundlegende Annahme des Modells lautet, dass durch die Beratung von Argumenten in einem öffentlichen Diskurs, der idealerweise unter Gleichen stattzufinden habe, eine höhere Legitimation erzielt werden könne als in rein machtbasierten Entscheidungsverfahren. ${ }^{14}$ Administrative Macht solle nicht nur aufgrund der gegebenen Verhältnisse ausgeübt werden, sie sei vielmehr fortlaufend diskursiv zu begründen. Hierfür bedarf es resonanzfähiger und autonomer Öffentlichkeiten. ${ }^{15}$ Das Modell fordert somit von den Beteiligten, dass sie gute Gründe für ihre eigenen Präferenzen angeben müssen - findet ein Akteur keine überzeugenden Argumente für sein eigenes Interesse, soll er im Rahmen einer ernsthaft geführten Argumentation die eigenen Präferenzen überdenken. Verhalten sich alle anderen Teilnehmer in diesem Sinne ebenfalls regelkonform, werden die Präferenzen schließlich auf der Grundlage von guten Argumenten in tragfähige, gemeinwohlorientierte Kompromisse transformiert. ${ }^{16}$ Dies bedeutet, dass politische Lösungen idealerweise am Ende eines längeren Diskurses gefunden werden, an dessen Verfahrensgestaltung entsprechend hohe Anforderungen zu stellen sind.

Im Mittelpunkt der Theorie der Deliberation steht der Gesetzgebungsprozess, da dort die gesellschaftliche Verständigung über die problematisierten, oft kontroversen Rechtsnormen erfolgt. ${ }^{17}$ Die Rechtsgemeinschaft wird somit als Assoziation freier und gleicher Bürger konzipiert, welche die Regeln ihres eigenen Zusammenlebens bestimmen. Um die Bürger

11 Vgl. z.B. Edana Beauvais und André Bächtiger, »Taking the Goals of Deliberation Seriously: A Differentiated View on Equality and Equity in Deliberative Designs and Processes «, (2016) 12 Journal of Public Deliberation.

12 Schmidt, Demokratietheorien (Fn. 10), 237.

13 Andreas Schäfer, Zwischen Repräsentation und Diskurs: Zur Rolle von Deliberation im parlamentarischen Entscheidungsprozess (Springer, 2017), 27.

14 Tim König, In guter Gesellschaft? Einführung in die politische Soziologie von Jürgen Habermas und Niklas Lubmann (Springer 2012), 13; Jon Elster, »Introduction«, in Jon Elster (Hrsg.), Deliberative Democracy (Cambridge University Press, 1998).

15 König, In guter Gesellschaft (Fn. 14), 22.

16 Ebd., 5-22.

17 Ebd., $12 \mathrm{ff}$. 
von andauernder Kommunikation zu entlasten, trifft das administrative System aber auch im Modell deliberativer Demokratie weiterhin die allgemein verbindlichen Entscheidungen - dies erfolgt allerdings idealerweise auf der Grundlage des vorangegangenen (und teilweise prozessbegleitenden) kommunikativen Prozesses. Das Konzept der deliberativen Demokratie ist expansionistisch angelegt: Mehr Partizipation führt demzufolge zu einer auch qualitativ besseren Demokratie.

Die Theorie zielt somit auf eine an Regeln und Verfahren gebundene Transformation von kommunikativer in administrative Macht. Allerdings ist das Idealmodell an äußerst anspruchsvolle Bedingungen geknüpft: ${ }^{18}$ Das »bessere« Argument soll in der Debatte zählen, Diskurse sollen frei von internen und externen Zwängen sein, und die Beteiligten sollen einen »Wir-Denkstil« pflegen, sich also nicht am eigenen, individuellen Nutzen orientieren, mithin nicht als egoistische Nutzenmaximierer auftreten. Auch bedarf es nachvollziehbarer, an diesen Kriterien ausgerichteter Verfahren, die es erlauben, die im Diskurs vorgebrachten Argumente zu bündeln und zu Positionen aufzubereiten, die im weiteren politischen Prozess einfacher "verarbeitet" werden können. Dies wiederum soll in einem Verfahren der Abwägung und des wechselseitigen Austauschs von Argumenten vorgenommen werden, nicht jedoch als rein statistische Aggregation, die sich an arithmetischen Mehrheiten orientiert. Die ausgetauschten Argumente sollen in der Debatte zudem verständlich bleiben, um keine Teilnehmer auszugrenzen; die Medien fungieren dabei idealerweise nicht als Macher, sondern als Vermittler von Argumenten. Im Ergebnis einer dergestalt angelegten Deliberation entsteht idealerweise ein dichtes Netz von demokratiefördernden Diskursen, das die Legitimation des politischen Prozesses zu festigen vermag.

\section{Chancen und Grenzen deliberativer Politik: Argumente der Beteiligungsforschung und institutionentheoretische Überlegungen}

Im Folgenden werden ausgewählte Argumente aus unterschiedlichen Forschungssträngen dargestellt: Neben den - hier kurz zusammengefassten - Argumenten der Beteiligungsforschung stehen im Wesentlichen institutionentheoretische Überlegungen im Vordergrund, die sich auf die Wechselwirkungen deliberativer Komponenten mit anderen Partizipationskanälen und dem Mehrebenensystem beziehen. Auf dieser Grundlage werden

18 Vgl. hierzu für viele Schmidt, Demokratietheorien (Fn. 10), $242 \mathrm{f}$. 
schließlich Schlussfolgerungen für die Nutzung deliberativer Elemente gezogen.

\subsection{Argumente der Beteiligungsforschung}

Theorien deliberativer Politik zufolge sollen freiwillige zivilgesellschaftliche Organisationen den Resonanzboden deliberativer Demokratie abgeben: Nicht die in sich vermachteten, an programmatischen Positionen orientierten und durchsetzungsorientierten Parteien und traditionellen Verbände, sondern zivilgesellschaftliche Akteure seien in der Lage, den Anforderungen eines Austauschs von Argumenten unter Gleichen zu genügen. Interessengruppen und Unternehmen sind von diesem Konzept der Zivilgesellschaft ausgeschlossen, weil diese Organisationen den Bürgerwillen manipulieren könnten. ${ }^{19}$ Der Theorie liegt somit ein eng gefasster, empirisch wenig unterfütterter Begriff der Zivilgesellschaft zugrunde. Die »Schattenseiten « zivilgesellschaftlicher Organisationen bleiben weitgehend unterbelichtet: Oft sind diese von staatlicher Finanzierung abhängig und somit nicht autonom, sie verfügen über eine geringe Zahl an Mitgliedern und sind gesellschaftlich schwach verankert. Ihre interne Organisation muss demokratischen Wahlen und durch diese begründeten Delegationsbeziehungen nicht genügen; vielmehr sind Tendenzen der Hierarchisierung und asymmetrische Machtbeziehungen innerhalb der Organisation oft unausweichlich. Die Beteiligung ist in zivilgesellschaftlichen Assoziationen sozial häufig höchst selektiv. Wie bei anderen Partizipationskanälen sind die gut ausgebildeten, ressourcenstarken Mittelschichten vertreten, die sich selbst ein hohes Maß an "Selbstwirksamkeit« zuschreiben, also davon ausgehen, dass sie ihre Präferenzen im politischen Prozess effektiv durchsetzen können. ${ }^{20}$ Einen ähnlichen Befund präsentieren auch Studien, die Fälle deliberativer Massenbeteiligung untersuchen; hier sind z.B. Frauen unterrepräsentiert und Amtsträger sprechen häufiger als »einfache» Bürger. ${ }^{21}$

Auch wenn die in den Foren ausgetauschten Argumente dem Idealmodell zufolge auch für Nicht-Experten verständlich bleiben müssen, so sind viele politische Materien doch von einer so erheblichen Komplexität ge-

19 König, In guter Gesellschaft (Fn. 14), 23.

20 Für die Schweiz vgl. für viele Wolf Linder, »Das politische System der Schweiz«, in Wolfgang Ismayr (Hrsg.), Die politischen Systeme Westeuropas (4. Aufl., VS, 2009).

21 Vgl. Gerber und Müller, »When the People Speak« (Fn. 6). 
prägt, dass die sachkundigen Bürger, die sich aktiv an einer Deliberation beteiligen, vor allem den gebildeten Mittelschichten entstammen. Dies dürfte vor allem dann der Fall sein, wenn - etwa bei infrastrukturellen Projekten - schwierige Fragen wie Gewässerschutz, geologische Fragen etc. verhandelt werden und es nicht mehr um das »Ob«, sondern um das konkrete »Wie« einer Planung und um Details der Durchführung geht, die allerdings oft politisch stark aufgeladen sind. Deliberative Foren müssen deshalb mit Bedacht zusammengesetzt werden, ansonsten vermögen auch sie ein zentrales Problem moderner Demokratien nicht zu beheben: die Bevölkerungsschichten, die mittlerweile an keiner Art der Beteiligung mehr teilhaben und sich aus dem politischen Diskurs zurückziehen, in den demokratischen Prozess zu reintegrieren. Sie dürften dieses Problem sogar noch verschärfen, sofern keine Vorkehrungen für die Zusammensetzung deliberativer Foren getroffen werden, die - z.B. über Losverfahren oder über eine Beachtung sozialer Merkmale - dafür Sorge tragen, dass unterschiedliche soziale Gruppen und Interessen angemessen repräsentiert sind und Gehör finden. Ist dies nicht gewährleistet, gerät deliberative Demokratie zu einer zusätzlichen Arena, die insbesondere die Gruppen bespielen können, die im politischen Prozess ohnedies bereits gut repräsentiert sind. Angesichts der Schwierigkeiten, alle Ziele der Deliberation gleichzeitig zu erreichen, plädieren empirische Untersuchungen dafür, den Prozess der Deliberation nicht unnötig zu belasten oder gleichzeitig alle idealisierten Ziele einlösen zu wollen: Praktiker sollten vielmehr Ziele priorisieren. Sie sollten also entscheiden, ob sie Aspekte der moral equality, die von konkreten sozialen Umständen abstrahieren, oder eher die der equity bei der Zusammensetzung von Foren beachten wollen. Letztere bezieht sich als Norm der Gerechtigkeit explizit auf die gegebenen sozialen Umstände. ${ }^{22}$

Da die Repräsentativität und Inklusivität deliberativer Foren häufig hinter den Erwartungen zurückbleiben, sind staatliche Akteure bzw. die »Designer " der Foren gefordert, die Auswahl der Teilnehmer zu bestimmen und geeignete Regeln zu setzen, um die typischen Defizite asymmetrischer Beteiligung auszugleichen. ${ }^{23}$ Schon geringfügige Veränderungen von Ab-

22 Beauvais und Bächtiger, »Taking the Goals of Deliberation Seriously« (Fn. 11).

23 Christoph Tewes, Claudia Saalbach und Ulrich Kohler, Bürgerbeteiligung bei umweltrelevanten Großprojekten: Der Beteiligungsbias als methodisches Instrument zur Bewertung von Beteiligungsverfahren (Uni Potsdam, 2014). Demzufolge sei eine gezielte Auswahl von Teilnehmern (auch bei Online-Verfahren) einer unkontrollierten Selbstselektion vorzuziehen, 48. 
stimmungs- und Verrechnungsregeln können die erzielten Ergebnisse fundamental verändern. ${ }^{24}$

Die Forschung über die Wirkung kognitiver Frames und von Shortcuts $^{25}$ fügt diesen Überlegungen eine weitere kritische Note hinzu. Studien zur politischen Psychologie belegen vielfach, dass Akteure sich insbesondere die Argumente zu Eigen machen, die mit ihren bereits festgefügten Positionen und Überzeugungen vereinbar sind oder die sie für »repräsentativ« für ein bestimmtes Problem halten. Andere Argumente werden von vornherein nicht erwogen oder ausgeblendet. Interessante empirische Einblicke bietet in diesem Zusammenhang das Mediationsverfahren zu "Stuttgart 21«, das als eines der umstrittensten Großprojekte in Deutschland gelten kann und bis heute zu erheblichen Protesten und gesellschaftlichen Verwerfungen geführt hat. Das Schlichtungsverfahren verdeutlicht, dass der Austausch von Argumenten ab einem bestimmten Zeitpunkt der Eskalation nur noch in begrenztem Umfang zu einer Präferenzänderung auf der Grundlage »besserer « Argumente führt. In einer Art experimenteller Anordnung wurden in einer Studie die gleichen 400 Probanden vor und nach dem Schlichtungsverfahren dahingehend befragt, inwiefern sie ihre Position angesichts des beobachteten Austauschs von Argumenten verändert hätten. ${ }^{26}$ Zwar gab ein großer Teil der Befragten an, im Rahmen der durchgeführten Mediation neue Argumente für oder gegen die Untertunnelung des Bahnhofs erfahren zu haben. Gleichwohl änderten die meisten befragten Personen ihre ursprüngliche, mittlerweile festgefügte Position zu diesem umstrittenen Projekt nicht mehr. Solche Erkenntnisse sprechen dafür, deliberative Elemente zu einem möglichst frühen Zeitpunkt des

24 Vgl. Schmidt, Demokratietheorien (Fn. 10), 248; Lucio Baccaro, André Bächtiger und Marion Delville, "Small Differences that Matter: The Impact of Discussion Modalities on Deliberative Outcomes«, (2014) 46 British Journal of Political Science 551. Die Autoren untersuchen in einer experimentellen Anordnung, wie unterschiedliche Verfahrensweisen, die Deliberation gestalten, den Outcome beeinflussen: Wenn Teilnehmer zu Beginn der Deliberation eine feste Position artikulieren, ändern sie ihre Meinung kaum noch, sie lernen jedoch stärker, und die deliberative Qualität steigt. Nehmen sie keine feste Position ein, fallen Lernprozesse und deliberative Qualität geringer aus, jedoch sind sie bereit, ihre Meinung zu ändern. Insofern bestehe ein Spannungsverhältnis zwischen deliberativer Verfahrensqualität und Meinungs- bzw. Positionswechsel.

25 Vgl. hierzu mit Beiträgen zu unterschiedlichen Aspekten: Thorsten Faas, Cornelia Frank und Harald Schoen, Politische Psychologie: Sonderheft 50 der Politischen Vierteljahresschrift (Nomos, 2015).

26 Frank Brettschneider, »Kommunikation und Meinungsbildung bei Großprojekten«, (2011) 61 Aus Politik und Zeitgeschichte 46. 
Policy-Prozesses einzusetzen. Ansonsten laufen sie insbesondere bei stark konfliktbehafteten Materien Gefahr, die ihnen zugeordnete argumentative Qualität nicht ausschöpfen zu können. Generell stellt sich bei dem hier für den Fall Stuttgart 21 geschilderten Untersuchungsdesign aber die Frage, ob und inwieweit die Öffentlichkeit, die an der Deliberation lediglich als Zuschauer beteiligt ist, ähnliche Lerneffekte erzielen kann wie die an der Deliberation unmittelbar beteiligten Akteure.

Im Zuge der zahlreichen Konflikte um Infrastrukturprojekte wurden in Deutschland die Verwaltungsverfahrensgesetze in Bund und Ländern dahingehend reformiert, dass die Bürgerschaft nun zu einem frühen Zeitpunkt von Entscheidungsverfahren - d.h. noch bevor die Agenda bestimmt wird - eingebunden werden soll. ${ }^{27}$ Für die Verwaltung bedeutet dies, dass sie sich stärker als bislang mit partizipationswilligen Bürgern bereits zu einem frühen Zeitpunkt der Planung auseinandersetzen muss. Doch auch unter diesen geänderten Bedingungen erfüllen "Anhörungen« der Bürgerschaft die Kriterien einer Deliberation zumeist nicht in dem Sinne, dass es zu einem Austausch von Argumenten unter »Gleichen« kommt.

Ferner ist die Annahme, Teilnehmer an einer Deliberation seien in der Lage, ihre Eigeninteressen zurückzustellen und sich auf die Kraft des guten Arguments einzulassen, in vielen Fällen zu idealistisch gedacht. Dagegen sprechen Überlegungen, welche die klare Trennung zwischen einem "Arguing ", das sich auf Sachargumente bezieht, und einem »Bargaining «, das individuelle Präferenzen zum Bezugspunkt strategischen Handelns nimmt, ${ }^{28}$ in Frage stellen. Argumente werden in Diskursen nicht nur zur Überzeugung des Gegenübers eingesetzt, sondern auch, um machtstrategische Vorteile zu erzielen. Die unterschiedliche argumentative Kraft von Akteuren kann asymmetrische Beziehungen zwischen den an deliberativen Foren teilnehmenden Personen weiter verfestigen. Mit anderen Worten: Machtpolitik beruht wesentlich nicht nur auf formalen Entscheidungskompetenzen und vorhandenen Asymmetrien, sondern auf der Durchschlagkraft von Argumenten, die verhandlungsstrategisch eingesetzt werden.

Kritisch könnte man ebenfalls einwenden, dass die Idee, gesellschaftliche Konflikte ließen sich mithilfe eines argumentativen, rationalen Verfah-

27 Verwaltungsverfahrensgesetz des Bundes, hier v.a. $\$ 25$, www.gesetze-im-internet.de/v wvfg/ (abgerufen am 22. November 2017).

28 Vgl. hierzu Jon Elster, "Arguing and Bargaining in Two Constituent Assemblies«, (1999) 2 Journal of Constitutional Law 345. 
rens und in einem Für und Wider mehr oder weniger harmonisch auflösen, umfassende gesellschaftliche Zielkonflikte zu verharmlosen droht. So lässt sich z.B. der bei großen Infrastrukturprojekten regelmäßig aufkeimende Streit über die Frage, ob wirtschaftlichem Wachstum oder ökologischen Zielen Priorität eingeräumt werden solle und wie die Folgen von Eingriffen in die Umwelt einzuschätzen sind, durch Argumente oft nicht befriedigend auflösen. Dies gilt auch für andere Grundsatzkonflikte, etwa über die Rolle des regulierenden Staates, die auf schwer vereinbare ökonomische Paradigmen zurückverweisen.

\subsection{Wechselwirkungen deliberativer Foren mit anderen Partizipationskanälen}

Würden Positionen und Argumente lediglich statistisch aggregiert, so wäre dies dem Charakter deliberativer Politik nicht angemessen. Vielmehr entfaltet diese Form der Partizipation die ihr zugedachten Wirkungen nur dann, wenn Argumente sorgfältig gegeneinander abgewogen und zu einer Entscheidungsgrundlage verdichtet werden. Dieses Erfordernis zieht spezifische Anforderungen an die Ausgestaltung der institutionellen Architektur nach sich, die in den Debatten über die Effekte deliberativer Elemente oft unberücksichtigt bleiben.

Unterliegen einzelne Gruppen im Austausch von Argumenten, sollten diese anschließend nicht mehr über die Möglichkeit verfügen, auf andere Handlungsarenen auszuweichen, in denen sie ihre eigenen Interessen durchsetzen können. In solchen Fällen würde das Legitimationspotenzial deliberativer Politik nachträglich entwertet. Dies wäre z.B. dann der Fall, wenn im Zuge einer Kombination von deliberativen und direktdemokratischen Elementen kollektive Akteure über die Möglichkeit verfügen, ihr Anliegen, das sie in einem deliberativen Forum nicht erfolgreich vertreten konnten, auf dem Weg eines Bürgerbegehrens und Bürgerentscheids erneut zu lancieren. Umgekehrt erhöhen plebiszitäre Verfahren aber ihrerseits den Druck, in einem frühen Stadium von Gesetzgebungsverfahren deliberative Elemente zu praktizieren. Anhand des Schweizerischen »Vernehmlassungsverfahrens « lässt sich etwa beobachten, dass sog. »referendumsfähige«, d.h. vetofähige, ressourcenstarke Gruppierungen früh in vorgeschaltete Beratungen einbezogen werden, um zu verhindern, dass 
diese anschließend auf die plebiszitäre Arena ausweichen..$^{29}$ Plebiszite und Verhandlungsforen sind in der Schweizer Demokratie eng aufeinander bezogen. ${ }^{30}$ Sofern diese, wie oft beschrieben, aber v.a. gut interessierte Interessengruppen und nicht zivilgesellschaftliche Organisationen einbinden, erfüllen auch sie die Anforderungen an eine »echte " Deliberation nur bedingt. Gegen eine solche Kombination verschiedener partizipativer Kanäle wird somit verschiedentlich kritisch eingewandt, dass sie vornehmlich die ohnedies durchsetzungsstarken Interessen begünstigt. Auch diese Beobachtung spricht dafür, dass staatliche Akteure - je nach Zielsetzung ${ }^{31}$ - die Zusammensetzung und Verfahren deliberativer Foren so regeln, dass auch den schwächeren, schlechter artikulierbaren Interessen ein Zugang verschafft werden kann.

Eine nahtlose Übertragung des Schweizer Modells, das von vielfältigen, ineinander verflochtenen Partizipationskanälen geprägt ist, auf parlamentarische Regierungssysteme ist kaum möglich. In fast allen europäischen Demokratien hängen die Regierungen vom Vertrauen ihrer parlamentarischen Mehrheiten ab, die sich nach Wahlen ebenso wie die parteipolitische Zusammensetzung der Kabinette verändern. Eine neu gewählte Regierung ist im parlamentarischen System Deutschlands deshalb z.B. sieht man von finanziellen Festlegungen und rechtlichen Bindungen $a b$ - weniger eng an die Entscheidungen ihrer Vorgänger gebunden als der Bundesrat in der Schweiz. Dort gibt es den engen Funktionszusammenhang zwischen Regierung und Regierungsmehrheit nicht. Der Schweizer Bundesrat ist vielmehr vom Prinzip der Konkordanz geprägt, das eine Repräsentation der wesentlichen Parteien in der Regierung vorsieht nicht zuletzt, um der Nutzung direktdemokratischer Instrumente durch die Opposition - gegen die Regierung - und damit potenziellen Regierungsblockaden vorzubeugen. Angesichts des nicht gegebenen Wechsels zwischen Regierung und Opposition nach Wahlen ist es eher möglich, eine größere Kontinuität in der Politikgestaltung herzustellen und somit auch Ergebnisse einer Deliberation weiter zu verfolgen. Richtet hingegen eine Regierung im parlamentarischen Regierungssystem, wie die deutsche Bundesregierung in der 18. Wahlperiode, deliberative Foren ein, um diskursiv Maßstäbe und Ziele eines "guten« Lebens zu ermitteln, so sind die

29 Zoltán T. Pállinger, »Direct Democracy in the Swiss Federation«, in Cristina Fraenkel-Haeberle et al. (Hrsg.), Citizen Participation in Multilevel Democracies (Brill, 2015).

30 Für den Schweizer Kanton Glarus vgl. Gerber und Müller, »When the People Speak« (Fn. 6).

31 Beauvais und Baechtiger, »Taking the Goals of Deliberation Seriously« (Fn. 11). 
daraus gewonnen Schwerpunktsetzungen für die nachfolgende Regierung politisch nicht bindend.

Wenn deliberative Foren und Dialoge ins Leben gerufen werden, bleibt häufig ungeklärt, welche Funktionen und Ziele sie im politischen Prozess denn eigentlich erfüllen sollen. Grundsätzlich sollen Deliberationsverfahren zu einer größeren Tolerenz abweichender Meinungen beitragen, Lerneffekte herbeiführen und Meinungen und Positionen verändern helfen. ${ }^{32}$ Inzwischen ist ein dichtes Netz solcher Foren entstanden, deren Rolle im Institutionensystem ebenso unscharf definiert ist wie ihre sinnvolle Verknüpfung mit anderen Partizipationskanälen. Angesichts der weit verbreiteten Forderung nach »mehr " (häufig direkter und partizipativer) Demokratie und der Verdrossenheit gegenüber repräsentativen Institutionen ${ }^{33}$ erhoffen sich politische Eliten von der bloßen Einrichtung solcher Diskussionsforen bereits einen Legitimationsgewinn. Die Foren werden jedoch häufig nicht sinnvoll mit vorhandenen Entscheidungsarenen verknüpft. Insofern gibt es kritische Stimmen, die einwenden, dass angesichts der beschriebenen Entwicklung der Entscheidungsprozess eher überlastet wird, als dass tragfähige, allseits akzeptierte Lösungen auf den Weg gebracht werden. ${ }^{34}$

Werden deliberative Foren top-down initiiert, mag dies mit Blick auf ihre repräsentative Zusammensetzung Vorteile haben. In einem von oben initiierten Verfahren können sie aber auch als Instrument der Regierung im politischen Prozess eingesetzt werden. Als illustrierendes Beispiel hierfür kann der Bayerische Energiedialog herangezogen werden, in dem über die Route der Stromnetztrassen beraten werden sollte. Die Planung von Netztrassen geht, wie die Vergangenheit vielfach gezeigt hat, typischerwei-

32 Fishkin und Luskin, »Experimenting with a Democratic Ideal« (Fn. 6); zu Tradeoffs zwischen den Zielen Beauvais und Baechtiger, »Taking the Goals of Deliberation Seriously« (Fn. 11).

33 Oscar W. Gabriel, »Repräsentationsschwächen und die zweite Transformation der Demokratie: Wer will in Deutschland direkte Demokratie?«, (2013) 44 Zeitschrift für Parlamentsfragen 592.

34 Pointiert hierzu E. Hien, «Mehr Bürgerbeteiligung - weniger Demokratie? Deutschland hat vor allem ein Problem mit der Durchsetzung bestandskräftiger Entscheidungen - und vergisst oft den rechtlichen Rahmen « Frankfurter Allgemeine Zeitung (Frankfurt, 24. Januar 2014), 7. Die Quintessenz dieses Beitrags lautet, dass Deutschland eher ein Implementations- denn ein Partizipationsproblem habe. 
se mit NIMBY-Problemen (»not in my backyard «) einher. ${ }^{35}$ Dies bedeutet, dass Bürger zwar die Energiewende grundsätzlich befürworten können, ihre erste Präferenz aber darauf gerichtet ist, eine unmittelbare räumliche Betroffenheit zu vermeiden. Die Bayerische Landesregierung schloss sich, obwohl sie 2011 die Energiewende und den Ausstieg aus der Kernenergie im Bundesrat mit unterstützt hatte, den Bürgerprotesten gegen den Trassenverlauf schließlich an. Angesichts der nahenden Kommunalwahlen war die Bayerische Landesregierung 2014 bestrebt, ihre Chancen im Wählerwettbewerb nicht zu schmälern. Sie bezog Stellung gegen den ursprünglich geplanten und bei den Betroffenen höchst umstrittenen Trassenverlauf, der durch Bayern führen sollte, wo die produzierende Industrie einen hohen Strombedarf hat. Sie forderte zunächst, dass eine für Bayern günstigere Regelung, der zufolge die Trasse erst weit im Süden in das Bundesland einbiegen sollte, realisiert wurde - dies hätte eine Planung zulasten anderer Regionen bedeutet. Angesichts des schwer auflösbaren Konflikts wurde schließlich einem unterirdischen Trassenverlauf der Vorzug gegeben, der jedoch mit deutlich höheren Kosten einhergeht.

Eine Auswertung von Stromnetzdialogen verdeutlicht ferner, dass die beteiligten Bürger sich oft nicht darüber im Klaren sind, welche Funktion den (häufig lokalen) Foren im politischen Entscheidungsprozess zukommt. In repräsentativen Demokratien können solche Foren nur eine beratende Funktion ausüben, denn sie sind schwächer legitimiert als die in allgemeinen, freien Wahlen bestimmten Volksvertreter und Parlamente. Deshalb vermögen sie parlamentarische Entscheidungen auch nicht zu ersetzen - auch wenn von ihnen in Einzelfällen eine faktische politische Bindekraft ausgehen kann. Demgegenüber sind die an Dialogen beteiligten Bürger häufig der Meinung, die Foren seien nicht konsultativer, sondern dezisiver Natur: Sie beharren somit konsequenterweise darauf, dass die ausgehandelten Ergebnisse die Entscheidungsträger inhaltlich binden müssten. ${ }^{36}$ Gewinnen die Bürger aber den Eindruck, ihre Beteiligung an den oft langwierigen und zeitaufwendigen Diskussionen laufe letztlich doch ins Leere, drohen deliberative Elemente die Legitimation der repräsentativen Demokratie aber eher zu vermindern als zu stärken.

35 Mit einer positiven Konnotation von NIMBY hingegen der Band von: Carol Hager und Mary A. Haddad (Hrsg.), Nimby Is Beautiful: Cases of Local Activism and Environmental Innovation around the World (Berghahn, 2015).

36 Christian Bauer, »Stiftung von Legitimation oder Politikverflechtungsfalle: Welche Folgen hat die Öffentlichkeitsbeteiligung beim Stromnetzausbau? « (2015) 8 Der moderne Staat 273. 
Werden deliberative Elemente zusätzlich zu anderen Beteiligungsformen genutzt, wirft dies zudem die Frage auf, wie die einzelnen partizipativen Komponenten zeitlich sinnvoll getaktet werden können. Deliberative Foren können im politischen Prozess eine beratende, befriedende und Entscheidungen begründende Wirkung haben, ihre Vertreter gehen aber nicht aus allgemeinen, freien Wahlen hervor. Foren und Dialoge sind in funktionierenden Demokratien legitimatorisch schwächer abgesichert als Parlamente. Nun soll deren Macht ebenso wie die der Ministerialbürokratien, die die Gesetze im Wesentlichen ausarbeiten, der Theorie zufolge durch Deliberation begründet und fortlaufend rückgekoppelt werden. Dies bedeutet, dass sich der Wert deliberativer Foren und von Dialogen vor allem dann entfalten kann, wenn diese ins Leben gerufen werden, bevor die Phase des Agenda-Settings abgeschlossen ist. Fühlen sich die Bürger hingegen mit einer Vorentscheidung konfrontiert, an der sie nur noch geringfügige Änderungen vornehmen können, dürfte dies ihr Misstrauen in die repräsentativen Institutionen eher verstärken. ${ }^{37}$ Ein »Talk « ohne »Action « entspricht jedenfalls nicht dem Grundgedanken deliberativer Demokratie. $^{38}$

Für Überlegungen, die sich auf Fragen der zeitlichen Taktung deliberativer Verfahren beziehen, bietet der Fall Stuttgart 21 erneut reichhaltiges Anschauungsmaterial. ${ }^{39}$ Das Schlichtungsverfahren wurde im Oktober 2010 ins Leben gerufen, nachdem mehr als 120 parlamentarische Anhörungen auf den unterschiedlichen Ebenen - Stadt, Land Baden-Württemberg und im Bund - stattgefunden und die Verwaltungsgerichte eine Vielzahl von Einsprüchen abgewiesen hatten. Die Proteste gegen die Untertunnelung des Stuttgarter Bahnhofs waren zu dieser Zeit bereits eskaliert, und die Positionen hatten sich weiter verhärtet. An der Schlichtung nahmen neben Vertretern der Deutschen Bahn AG und der Landesregierung u.a. die Vertreter von gesellschaftlichen Initiativen teil, die sich im Laufe des

37 Vgl. Hans-Jörg Birk, »Frühe Bürgerbeteiligung in Planungsverfahren«, (2011) 30 Neue Juristische Wochenschrift; Alexander Schink, „Öffentlichkeitsbeteiligung - Beschleunigung - Akzeptanz: Vorschläge zur Verbesserung der Akzeptanz von Großprojekten durch Öffentlichkeitsbeteiligung«, (2011) 22 Deutsches Verwaltungsblatt 1377.

38 König, In guter Gesellschaft, (Fn. 14), 18, mit Verweis auf: Nils Brunsson, The Organization of Hypocrisy: Talk, Decisions and Actions in Organizations (Wiley, 1989).

39 Vgl. Sabine Kropp: »Runderneuerung der repräsentativen Demokratie im Bundesstaat oder: Welche Lehren ziehen wir aus Stuttgart 21?«, in Silke I. Keil und S. Isabell Thaidigsmann (Hrsg.), Zivile Bürgergesellschaft und Demokratie. Aktuelle Ergebnisse der empirischen Politikforschung. Festschrift für Oscar W. Gabriel zum 65. Geburtstag (VS, 2012), 469-85. 
langjährigen Protests formiert hatten. ${ }^{40}$ Es hatte zuvor ein klares Votum der Parlamentsmehrheiten auf allen Ebenen für die Untertunnelung und den Ausbau gegeben; die Vertreter verfügten in der Schlichtung über keine Entscheidungskompetenz. Damit begab man sich unter Gesichtspunkten demokratischer Legitimation auf ein rutschiges Gelände, da die Erwartung geschürt wurde, die Ergebnisse der Deliberation seien parlamentarischen Entscheidungen legitimatorisch übergeordnet und ggf. dezisiver $\mathrm{Na}$ tur. Unklar blieb indessen, ob am Ende der Faktenschlichtung ein Schlichterspruch stehen und wie verbindlich dieser sein sollte, und ob sich dieser auf das weitere Verfahren oder auch auf konkrete Inhalte zu beziehen hatte. Verfahrensunklarheit, mangelnde Ergebnisoffenheit und ein enges Zeitfenster prägten das Schlichtungsverfahren. Dessen Bezug zu den parlamentarischen Verfahren blieb ungeklärt, und die Ergebnisse wurden von den Gegnern des Projekts schließlich auch nicht akzeptiert.

Der Schlichterspruch trug somit auch nicht zu einer höheren Legitimation der vorgeschlagenen Lösung bei. Der anschließende Volksentscheid, bei dem die Kofinanzierung des Landes Baden-Württemberg zur Entscheidung stand, verfügte über eine höhere formale Legitimation als das deliberative Verfahren, das im Idealfall einem Volksentscheid vorgeordnet sein sollte ${ }^{41}$ - wobei letzterer eben auch andere Ergebnisse als die Deliberation hervorbringen kann. Wie oben bereits gezeigt, sind spät eingesetzte Schlichtungsverfahren nur noch bedingt geeignet, um auf der Grundlage eines offen geführten Für und Wider Positionswechsel der Beteiligten herbeizuführen - vielmehr werden solche Debatten typischerweise eher positionsbezogen geführt. Der Fall Stuttgart 21 stützt somit abermals das hier vorgebrachte Argument, dass deliberative Foren ihre positive Wirkung nur dann entfalten können, wenn sie zu einem möglichst frühen Zeitpunkt im Policy-Prozess eingesetzt werden und über hinreichend Zeit verfügen, um einen fairen Austausch von Argumenten zu ermöglichen. Zur faktischen nachträglichen Korrektur parlamentarischer Entscheidungen eignen sie sich indessen nicht - weder in legitimatorischer Hinsicht noch aus Verfahrensgründen.

40 Vgl. Schlichtung S21, „Die Teilnehmerinnen und Teilnehmer bei den Schlichtungsgesprächen«, (2010) www.schlichtung-s21.de/teilnehmer.html (abgerufen am 22. November 2017).

41 Gerber und Müller, »When the People Speak« (Fn. 6). 


\subsection{Deliberative Politik im Mehrebenensystem}

Mehrebenensysteme bieten den Bürgern tendenziell mehr Möglichkeiten, an Wahlen und Abstimmungen zu partizipieren, als unitarische Systeme. Ihre Entscheidungsverfahren sind angesichts fragmentierter, verflochtener und sich überlappender Institutionen aber auch komplizierter. Aus dieser Komplexität ergeben sich bestimmte Herausforderungen an die Nutzung zusätzlicher partizipativer Komponenten. Da deliberative Foren keine Entscheidungsfunktion ausüben, können sie - ohne dass bestehende Zuständigkeiten umfassend verändert werden müssen - in die Gesetzgebungsverfahren auf den unterschiedlichen Ebenen eines Mehrebenensystems einpasst werden. Jedoch sollten nicht nur ihre Wechselwirkungen mit den bestehenden repräsentativen Institutionen abgewogen werden, sondern auch ihr Einfluss auf Entscheidungen, deren Wirkung über eine territorial verfasste Jurisdiktion hinausreicht. Folgeabschätzungen, wie sich die auf lokaler oder regionaler Ebene praktizierte partizipative Demokratie auswirken könnte, und wie mit sog. "Spill-Overs « umzugehen ist, sind somit erforderlich, will man nicht-intendierte Effekte vermeiden. Solche Effekte werden in (föderalen) Mehrebenensystemen typischerweise in intergouvernementalen Verhandlungen ausgeglichen, die sich der unmittelbaren Partizipation der Bürger entziehen. ${ }^{42}$

Insbesondere wenn Probleme die Grenzen territorial verfasster Jurisdiktionen überschreiten, sind deliberative Foren, die auf lokaler oder regionaler Ebene angesiedelt sind, selten in der Lage, Lösungen herbeizuführen, die die Interessen unterschiedlicher territorialer Einheiten ausgleichen. Um externe Effekte internalisieren zu können, bedürfen sie einer Aggregation mit den Präferenzen anderer Gebietseinheiten durch repräsentative Institutionen. Diese sollten die Ergebnisse und Argumente lokaler Foren zwar berücksichtigen, sie können sie aber im Falle von Externalitäten nicht nahtlos umsetzen, ohne ihrerseits defizitäre öffentliche Güter zu produzieren.

Ungeachtet der Frage, wie deliberative Elemente im Einzelnen auszugestalten und welche Anforderungen dabei zu beachten sind, scheinen sich eher solche Materien für eine Deliberation zu eignen, die zu den ausschließlichen Zuständigkeiten einer Ebene zählen. Sind die Zuständigkei-

42 Vgl. aber die Versuche der Demokratisierung von exekutiven Verhandlungen in Kanada: Julie Simmons, »Democratizing Executive Federalism«, in Herman Bakvis und Grace Skogstad (Hrsg.), Canadian Federalism: Performance, Effectiveness and Legitimacy (3. Aufl., OUP, 2011). 
ten der territorialen Einheiten eindeutig getrennt und bleiben Problemlagen auf lokale oder regionale Räume begrenzt, können Deliberationen unabhängig voneinander in den einzelnen Jurisdiktionen des Mehrebenensystems stattfinden. Schwieriger gestaltet sich der Einbau von deliberativen Elementen hingegen, wenn Problemlagen die Grenzen einer Einheit überschreiten und Externalitäten in intergouvernementalen Verhandlungen, also durch Regierungen und Verwaltungen, ausgeglichen werden müssen. In solchen Fällen müssen die Exekutiven die Präferenzen, die sich durch die Erörterung von Argumenten in den deliberativen Foren der jeweiligen Gebietseinheiten herauskristallisiert haben, abermals gegeneinander abwägen und ggf. verändern. Gute Argumente können dann zwar aufgegriffen werden, sie werden jedoch in intergouvernementalen Verhandlungen, in denen territoriale, parteipolitische und sektorale Interessen ausgeglichen werden müssen, notgedrungen weiter zu Kompromissen verarbeitet. Die an sich naheliegende Idee, deliberative Foren so auszugestalten, dass sie ihrerseits die Mehrebenenstruktur abbilden, stößt auf Grenzen der Praktikabilität: Solche Foren müssten zahlreiche Akteure, die ihrerseits eine Vielzahl von Interessen repräsentieren, umfassen. Die Meinungsbildung würde sich äußerst komplex gestalten. ${ }^{43}$ Dass Bürger und zivilgesellschaftliche Organisationen ihrerseits die Mechanismen von Kompromissen und des Ausgleichs, die typisch sind für intergouvernementale Verhandlungen, in die Abwägung eigener Argumenten vorab einbeziehen, ist eine äußerst voraussetzungsvolle Annahme, die sich in der Realität kaum einlösen lässt.

Die angestellten Überlegungen weisen dennoch darauf hin, dass deliberative Komponenten mit politikverflochtenen Mehrebenensystemen, in denen die Gebietseinheiten Programme gemeinsam entscheiden und finanzieren, besser kompatibel sind als eine Volksgesetzgebung. Über deren Einführung auf Bundesebene wird - in Anlehnung an die Schweizer Erfahrungen - in Deutschland seit Jahren intensiv diskutiert. Eine Volksgesetzgebung ist dezisiv, was bei Kompetenzen, die von Bund und Ländern gemeinsam ausgeübt werden, z.B. die Frage aufwirft, ob ein Landeselektorat auf solche Materien per Volksentscheid zugreifen können sollte oder nicht - und wer bei politikverflochtenen Materien, in denen Bund und Länder gemeinsam entscheiden, das Elektorat sein soll. Der Schweizer Föderalismus, in dem die Zuständigkeiten zwischen den Ebenen trotz zahlreicher Kooperationsformen eindeutiger getrennt wird, kennt solche engen Verflechtungen nicht. Dort können die Kantone z.B. auch aus den

43 Vgl. ebd. 
sog. "Konkordaten« aussteigen, so dass diese Vereinbarungen nicht alle Gebietskörperschaften umfassen müssen.

Das Beispiel Stuttgart 21 liefert auch für diesen Aspekt Anschauungsmaterial. Der 2011, also nach dem Schlichtungsverfahren durchgeführte Volksentscheid in Baden-Württemberg kann zwar unter formalen Gesichtspunkten als unproblematisch gelten, weil darin von den Stimmbürgern lediglich über den freiwilligen Anteil des Landes an der Bahnhofsfinanzierung - und damit über eine reine Landeszuständigkeit - entschieden wurde. Bekanntlich hat sich die Mehrheit der Abstimmenden am 27. November 2017 gegen den Ausstieg entschieden; die Kritiker verfehlten auch das Quorum von 33 Prozent der Wahlberechtigten. Faktisch jedoch wäre der politische Druck bei einem Votum zugunsten des Ausstiegs aus der Landesfinanzierung so groß gewesen, dass das Projekt, dem eine überregionale Bedeutung für die Planung der Verkehrsinfrastruktur zukommt, insgesamt nicht länger zu halten gewesen wäre. ${ }^{44}$

\section{Was kann deliberative Demokratie bewirken?}

Die vorangestellten Überlegungen zeigen, dass durch deliberative Demokratie bessere Argumente für politische Entscheidungen gewonnen werden können und deren Akzeptanz somit gesteigert werden kann. Mehr Partizipation muss aber nicht automatisch zu mehr oder einer »besseren« Demokratie führen. Werden zusätzliche Partizipationselemente ohne Wechselwirkungen zu bedenken und ohne dass Verfahrensklarheit oder Ergebnisoffenheit besteht in ein Institutionengefüge eingebunden, kann dies die Repräsentativität vertretener Positionen weiter verzerren. Die oben präsentierten Argumente legen nahe, dass der Mehrwert deliberativer Foren begrenzt bleibt, wenn diese zu zahlreich sind und eingesetzt werden, ohne dass zuvor genau geklärt wird, welche Ziele ihnen im Entscheidungs- bzw. Gesetzgebungsprozess zugedacht werden. Bindet man Bürger und Zivilgesellschaft in das vorparlamentarische Stadium von Gesetzgebungsprozessen ein, sollte ihnen die - zuweilen begrenzte - Bindekraft der

44 An dieser Stelle ließe sich einwenden, dass auch im Falle der Politikverflechtung die einzelnen Parlamente häufig die letzte Entscheidungsbefugnis haben und sie somit theoretisch in der Lage sind, intergouvernemental ausgehandelte Kompromisse im Zuge einer ex post-Kontrolle aufzuschnüren. Angesichts des in parlamentarischen Systemen bestehenden Legitimationszusammenhangs, in dem die in intergouvernementalen Verhandlungen vertretenen Exekutiven vom Vertrauen ihrer Parlamentsmehrheiten abhängen, tritt dieser Fall jedoch äußerst selten ein. 
von ihnen erarbeiteten Vorschläge bewusst sein, um Missverständnissen vorzubeugen. Ansonsten kann der Eindruck entstehen, es werde lediglich die Machtlosigkeit der Bürger demokratisiert und partizipative Verfahren dienten zuvorderst dazu, den Eliten eine zusätzliche, vordergründige Legitimationsquelle zu verschaffen.

Deliberationen lassen sich eher in überschaubaren Territorien, weniger jedoch im nationalen Maßstab durchführen. ${ }^{45}$ Generell scheinen in komplexen institutionellen Umgebungen Materien, deren Wirkungskreis lokal und regional begrenzt ist, besser geeignet als solche, die die Grenzen von Jurisdiktionen überschreiten oder von mehreren Jurisdiktionen gemeinsam entschieden und finanziert werden. $\mathrm{Zu}$ diskutieren wäre auch, ob sich die Tugenden deliberativer Demokratie eher entfalten können, wenn die beteiligten Akteure die Auswirkungen ihrer Beratschlagung auf ihre individuelle Lebenslage nicht exakt bestimmen können. Sie werden sich in diesem Fall stärker um eine "gerechte « Lösung bemühen, die auch die Interessen anderer Beteiligter einschließt. ${ }^{46}$ Dieses Argument spräche dafür, dass sich insbesondere solche Themen eher für eine Deliberation eignen, die von einer gewissen Abstraktheit geprägt sind. Hierzu zählen z.B. Reformen der Verfassung, mit denen Bürger die Grundlagen ihres Zusammenlebens regeln. Aber auch Faktenschlichtungen und die Durchführung von Sachdebatten sind in kleinerem Rahmen durchaus möglich, wobei deren Erfolg - wie nicht zuletzt das Beispiel Stuttgart 21 zeigt - an eine Vielzahl von Voraussetzungen gebunden ist.

Die Theorie der Deliberation setzt hohe Anforderungen an die Rationalität der Teilnehmer. Sie blendet aus, dass Sprache und Kommunikation nicht notwendig konsensstiftend wirken, sondern andere Beteiligte auch ausgrenzen können. ${ }^{47}$ Sachargumente werden häufig strategisch eingesetzt und können die oft gegebene asymmetrische Machtverteilung zugunsten ressourcenstarker Akteure innerhalb deliberativer Foren festigen. Nicht unproblematisch erscheint auch die in der Theorie vorgenommene normative Differenzierung von traditionellen kollektiven Akteuren, wie Parteien und Verbänden, und einer demgegenüber positiv konnotierten Zivilgesellschaft, der per se schwächer ausgeprägte egoistische Verhaltensweisen und eine stärkere Gemeinwohlorientierung zugeordnet werden.

45 Gerber und Müller, »When the People Speak« (Fn. 6).

46 Im Sinne eines »Schleiers des Nichtwissens«, vgl. John Rawls, Eine Theorie der Gerechtigkeit (Suhrkamp, 1993).

47 Vgl. Schmidt, Demokratietheorien (Fn. 10), 249. 
Insgesamt bleibt festzuhalten, dass die Theorie erfahrungswissenschaftlich besser abgesichert werden muss. Vor dem Hintergrund der oben erwähnten Einwände und Befunde liegt es nahe, deliberative Demokatie eher als eine Art regulativer Idee zu begreifen. Die in der Theorie formulierten Ansprüche sind so voraussetzungsvoll, dass sie nur mit Abstrichen empirisch umgesetzt werden können. ${ }^{48}$ Deliberative Demokratie wäre insofern nach Habermas als ein rekonstruktives Verfahren zu begreifen, das die engen Grenzen der Erfahrungswissenschaft aufbricht. Rationale Verfahren können dabei jenseits des empirisch unmittelbar Erfahrbaren verdeutlicht werden, ohne dass empirische Grenzen aus den Augen verloren würden. ${ }^{49}$ Somit wäre es verfehlt, das ganze Konzept deliberativer Demokratie nur deshalb zu verwerfen, weil nicht alle Kriterien von den real existierenden Fällen eingelöst werden können: »Mit der Umdefinition von normativen Anforderungen an offene demokratische Meinungsund Willensbildungsprozesse in erwünschte Konsequenzen politischer Entscheidungen wird auch der empirischen Deliberationsforschung eine verengte und einseitige Perspektive zugewiesen. ${ }^{50}$ Empirisch informierte Studien müssen sich deshalb darum bemühen, die Fallstricke, die sich aus einer möglichen verzerrten Repräsentation und den geschilderten institutionellen Spannungslagen ergeben können, mit zu bedenken, damit die demokratietheoretischen Ziele dieser partizipativen Verfahren nicht verloren gehen oder gar in ihr Gegenteil umschlagen.

48 Beauvais und Baechtiger, »Taking the Goals of Deliberation Seriously« (Fn. 11); Baccaro, Bächtiger und Deville, »Small Differences that Matter« (Fn. 24).

49 Vgl. Frank Nullmeier, »Intersubjektivität und Interindividualität: Anmerkungen zu den sozialtheoretischen Grundlagen der Demokratietheorie«, in Flügel-Martinsen et al. (Hrsg.), Deliberative Kritik (Fn. 8), 83.

50 Vgl. Thomas Saretzki, »Deliberative Politik und demokratische Legitimität: Perspektiven der Kritik zwischen empirischer Deliberationsforschung und reflexiver Demokratie«, Flügel-Martinsen et al. (Hrsg.), Deliberative Kritik (Fn. 8), 45f. 